\title{
Using coordinate-based meta-analyses to explore structural Imaging Genetics
}

Hildegard Janouschek, MD'1)*; Claudia R. Eickhoff(2),3),4)*, MD; Thomas W. Mühleisen, PhD $^{3), 5)}$;

Simon B. Eickhoff, MD ${ }^{3), 6)}$; Thomas Nickl-Jockschat, $M D^{2), 4}$

1) Department of Neurology, RWTH Aachen University, Aachen, Germany

2) Department of Psychiatry, Psychotherapy and Psychosomatics, RWTH Aachen University, Aachen, Germany

3) Institute of Neuroscience und Medicine (INM-1), Research Centre Jülich, Jülich, Germany

4) Jülich-Aachen Research Alliance Brain, Jülich/Aachen

5) Department of Biomedicine, University of Basel, Basel, Switzerland

6) Institute of Clinical Neuroscience and Medical Psychology, Heinrich Heine University, Düsseldorf, Germany

* both authors contributed equally

\section{Corresponding author:}

Prof. Dr. Thomas Nickl-Jockschat

Department of Psychiatry, Psychotherapy and Psychosomatics, RWTH Aachen University

Pauwelsstrasse 30, D-52074 Aachen, Germany

Mail: tnickl-jockschat@ukaachen.de

Word count: 5492

Running title: Using meta-analyses in Imaging Genetics

Number of tables: 2

Number of figures: 1

Number of supplementary material: 2 supplementary tables 


\section{Abstract}

Imaging genetics has become a highly popular approach in the field of schizophrenia research. A frequently reported finding is that effects from common genetic variation are associated with a schizophrenia-related structural endophenotype. Genetic contributions to a structural endophenotype may be easier to delineate, when referring to biological rather than diagnostic criteria. We used coordinate-based meta-analyses, namely the anatomical likelihood estimation (ALE) algorithm on 30 schizophrenia-related imaging genetics studies, representing 44 single-nucleotide polymorphisms at 26 gene loci investigated in 4,682 subjects variants. To test whether analyses based on biological information would improve the convergence of results, gene ontology (GO) terms were used to group the findings from the published studies. We did not find any significant results for the main contrast. However, our analysis enrolling studies on genotype $X$ diagnosis-interaction yielded two clusters in the left temporal lobe and the medial orbitofrontal cortex. All other subanalyses did not yield any significant results. To gain insight into possible biological relationships between the genes implicated by these clusters, we mapped five of them to GO terms of the category "biological process" (AKT1, CNNM2, DISC1, DTNBP1, VAV3), then five to "cellular component" terms (AKT1, CNNM2, DISC1, DTNBP1, VAV3), and three to "molecular function" terms (AKT1, VAV3, ZNF804A). A subsequent cluster analysis identified representative, non-redundant subsets of semantically similar terms that aided a further interpretation. We regard this approach as a new option to systematically explore the richness of the literature in imaging genetics.

\section{Introduction}


Since Egan and colleagues (Egan et al. 2001) published their iconic paper on differential working memory-related neural activations due to the Val158Met polymorphism in the dopamine catabolism-related COMT gene (rs4680), the field of imaging genetics has become a highly popular approach in schizophrenia research. Motivated by the hypothesis that gene variants exert stronger effects on a neuroimaging endophenotype than on a behavioural phenotype (Mier et al. 2010), the characterization of functional and structural neuroimaging correlates associated with genetic variation has been pursued with the intention to bridge the gap between molecular genetics and neural systems level findings.

Classical approaches with candidate genes, however, have met important obstacles. Most common variants associated with psychiatric disorders such as schizophrenia only exhibit low effect sizes regarding the manifestation of the disorder [3]. Thus, imaging genetics studies are necessarily based on the hypothesis that effects on a neuroimaging endophenotype are extensively greater than on a behavioral level to get reliably detected with the usual sample sizes of a few hundred individuals ${ }^{2}$. However, if this assumption does not hold true, the option to identify effects on brain structure and/or function associated with these genetic variants by imaging genetics approaches will be severely hampered. Meta-analyses should be able to identify brain regions, whose structure or function is consistently associated with a particular genetic variant across individual studies. Though, a recent coordinate based meta-analysis on working memory-related neural activations and the rs4680 single-nucleotide polymorphism (SNP) - the most extensively studied gene-brain function association today - did not retrieve significant results [4]. Although a variety of confounds might have influenced this negative finding, it should be emphasized that a meta-analytic validation of the imaging genetics concept still is lacking, at least at a whole-brain level.

Given these limitations, a quantitative review of the existing literature on imaging genetics with coordinate-based meta-analyses $[5,6]$ has the potential to overcome the shortcomings of the individual study. This type of meta-analysis determines, whether the reported maxima spatially converge above chance across individual studies. Thus, it provides a tool to capitalize on the wealth of the published literature and opens up new opportunities to systematically explore the 
existing data. In the context of imaging genetics, this concept can be applied in at least two meaningful ways: first, it can serve to investigate, whether the effects of one given gene variant on brain structure and/or function spatially converge in distinct brain regions above chance. This approach has been demonstrated previously by our lab in a study on neural activations related to working memory tasks and the COMT Val158Met (=rs4640) polymorphism [4]. Second, it can be used to explore, whether different gene variants converge with their effects in the same brain regions above chance. In other words: if there is a claim in the literature that different gene variants all exert their effects, e.g., in the frontal lobe, coordinate-based metaanalysis is a powerful tool to determine, whether this claim actually holds true.

This latter approach has the great advantage that it is able to corroborate the contributions of different gene variants to a given endophenotype. In contrast to genome-wide association studies that use a structural (or functional) endophenotype for gene discovery [7] the actually presented approach comes with the disadvantage that it is certainly limited to - and, therefore, biased accordingly - the gene variants, on which studies have already been conducted. On the other hand, it goes along with three comparative advantages: it allows the pooling over large cohorts largely without any additional costs. It seems also more sensitive in comparison to genome-wide approaches, since the number of genes is already narrowed down by different a priori-hypotheses. Finally, this approach also allows exploratory analyses, whether, e.g., a grouping of studies based on biological information of the genes or on diagnostic criteria is more likely to yield significant results.

Imaging genetics studies on risk gene variants that have been reported to be associated with a schizophrenia-related brain endophenotype are good candidates in this regard. One of the most outstanding findings that was reported across many original studies is that structural changes associated with a putative risk allele were predominantly found in regions associated with the disorder, irrespective of the nearest gene to which the variant maps [8-12]. These susceptibility regions seemed to be affected by schizophrenia risk variants, regardless whether schizophrenia patients or healthy controls were examined [10, 13-15]. These reports seem even more striking, when taken into consideration that some of the examined gene variants 
that were initially studied as schizophrenia susceptibility candidates were not confirmed in genome-wide association studies (GWAS) or even with substantial evidence against a role in the pathogenesis of the disorder (e.g. DISC1 [16] and COMT [17]). Consequently, gene variants might contribute to a neuroanatomical endophenotype, while they are not involved in the pathogenesis of the disorder itself [18].

We, therefore, decided to conduct a meta-analysis that pools over all available studies that claim to study risk genes associated with a structural schizophrenia-related endophenotype

Coordinate-based meta-analysis is a powerful tool to determine, whether this notion of a clustering in schizophrenia susceptiblity regions can be objectively reproduced by synthesizing the results of the individual studies. It has also the potential to explore, whether potentially convergent findings are largely driven by schizophrenia variants identified by GWAS or whether there is little overlap.

Moreover, coordinate-based meta-analysis of structural imaging genetics studies could also provide important hints to another central riddle of schizophrenia. The currently used diagnostic classification symptoms define schizophrenia as a syndrome, with its diagnosis based on a constellation of clinical symptoms, rather than a common pathomechanism $[19,20]$. Thus, the concept of schizophrenia might serve as an umbrella, under which various etiologically and pathophysiologically diverse disease entities are subsumed [21]. This notion has led to the claim that schizophrenia is not a valid concept for biological research and should be "deconstructed" $[22,23]$ or even completely abolished also for clinical use. Congruent with those ideas, schizophrenia patients usually exhibit high interindividual variance with regard to, e.g., brain structure changes, reflecting a higher heterogeneity amongst the patients than amongst healthy controls [22,24]. This heterogeneity in the patient group has been explained due to different patterns of genetic lesions in individual patients that, in turn, lead to different endophenotypes, which are phenotypically, however, all assumed under the diagnosis of schizophrenia [22]. Given these considerations, if endophenotypes can serve as markers for 
increased vulnerability also in healthy risk genotype carriers, then these endophenotypes might be easier to delineate, when recurring to biological criteria rather than diagnosis.

The use of biological criteria should, therefore, help to reduce heterogeneity of findings. Following this line of thought, pooling meta-analytically over data sets of structural imaging genetics studies can be expected to reduce biological heterogeneity more and, therefore, yield more likely significant results, when studies are grouped by biological criteria, i.e., according to pathways or biological functions of the studied genes, than by diagnostic criteria, e.g., whether patients or healthy controls were enrolled in an actual study. This approach is based on the assumption that variants in genes that are involved in common biological processes are more likely to cause structural changes in identical brain regions than those variants that are not part of a joint biological mechanism. While the explanatory power of a meta-analysis on classical imaging genetics studies - unlike GWAS-based analyses of original neuroimaging data sets - is certainly limited to the set of gene variants that were reported in the respective publications, an increased homogeneity of findings due to the application of biological or diagnostic criteria nevertheless has the potential to add important clues to this central puzzle of schizophrenia. Coordinate-based meta-analysis, therefore, provides a powerful tool to quantitatively test such hypotheses based on the spatial convergence of imaging genetics findings capitalizing on the richness of the existing literature. 


\section{Methods}

\section{$\underline{\text { Literature Search and Selection }}$}

We performed a literature search for structural magnetic resonance imaging (sMRI) studies using voxel-based morphometry (VBM) to determine gene $\mathrm{X}$ structure interactions of genetic variants that the authors suspected to cause a schizophrenia-relevant endophenotype, using Pubmed (www.pubmed.org, search strings in Supplementary Table 1) and Google Scholar (http://scholar.google.de/, search strings "Schizophrenia and [risk genes or risk gene] or [SNP or single nucleotide polymorphism] and whole brain and [voxel based morphometry or vbm]“. Additional papers were then identified through reference tracing from the retrieved articles as well as from qualitative reviews of the literature. To avoid an early selection bias due to overtly restrictive inclusion criteria, we decided to include all studies that claimed to investigate genetic markers that might contribute to a schizophrenia-relevant endophenotype. In a subsequent step, the respective gene variants were classified according to their evidence for an association with the disorder itself (see Table 1).

The inclusion criteria were as follows: (1) structural MRI studies listed on Pubmed or Google Scholar that have been published until June 3rd, 2015, (2) original peer-reviewed studies, (3) whole brain assessment not restricting analysis or inference to a priori regions of interest, (4) studies reporting contrasts due to genotypes of susceptibility gene variants that might contribute to a schizophrenia-relevant endophenotype, if studies reported also contrasts due to genotypes of susceptibility markers for other disorders, only those coordinates were included in the analysis that were described as relevant for schizophrenia, (5) complete reporting of peak coordinates of all clusters in stereotactic space (Talairach/MNI), and (6) bidirectional analysis of contrasts (i.e. analysis of both GM increases and decreases).

We extracted all genes that were assigned to common polymorphisms by the original publications. Symbols of the reported genes were checked if they match to the latest approved definition by the Human Gene Nomenclature Committee (HGNC; www.genenames.org) [23]. Disapproved symbols were substituted by the approved symbols (Supplementary Table 2). 
Our search identified a total of 358 articles in Pubmed or Google Scholar. 328 studies had to be excluded due to one of the following reasons: no whole brain data were available, no stereotactic coordinates available, no direct linear contrasts according to genotype, studies contained solely data derived from ROI-based analyses. A total of 30 studies were included in the meta-analysis that enroll 4,682 subjects $(3,081$ healthy controls, 1,356 schizophrenia patients, 52 patients with bipolar disorder, 37 patients with OCD, a sample consisting of 65 high risk individuals with or without symptoms, 43 SNPs and one length polymorphism located at 26 gene loci (Table 1).

\section{Categorization of studies according to the original contrasts}

The selected studies were categorized due to the original contrasts for which VBM coordinates were reported. Imaging and genetic data were matched to the most frequently reported categories as follows: genotype effects, gene $\mathrm{x}$ diagnosis interactions, gene $\mathrm{x}$ gene interactions, gender $x$ genotype and age $x$ genotype interactions. Each of the reported coordinates was then tagged with the fitting category serving as a label for the meta-analyses. Only categories providing a sufficient number of experiments $(n \geq 15)$ were used for further analyses. In detail, these were the categories genotype effects and genotype $X$ diagnosis interactions. The included coordinates were tagged with the following information from the original publication: study name, marker name (if more than one marker was reported, coordinates were tagged by the markers that were studied in the respective contrast), and gene symbol.

\section{Categorization of studies according to gene ontology}

The functional categories of gene ontology (GO) describe gene products with regard to their associated „biological processes“, „cellular components“, and „molecular functions”, in a species-independent manner. The selected gene symbols were mapped to GO terms and 
tested for an enrichment of gene sets (GSEA) in the three functional categories using a tool from the STRING database version 10.0 (http://string-db.org/) with the configurations "genome background", "specific", "IEA" (inferred from electronic annotation), and "Bonferroni" (correction for multiple comparisons) [24]. The GSEA was performed for each category separately. The top 5 enriched GO terms of each category were included in the subsequent meta-analysis for which the underlying genetic markers provided a sufficient number of VBM experiments ( $\mathrm{n} \geq 15)$. These were the terms "Axon", "cellular prohection", "neuron part", "neuron projection", "somatodendritic compartment" and "regulation of protein modification process".

\section{$\underline{\text { Condensation of gene ontology terms }}$}

To sum up and visualize the biological information provided by the identified GO terms, the tool REduce + VIsualize Gene Ontology (REVIGO; http://revigo.irb.hr) was used [25]. The procedure is based on the concept of semantic similarity measures [26]. For summarization of the terms, REVIGO performs a clustering analysis that is similar to hierarchical clustering methods such as the neighbor joining approach. REVIGO prioritizes terms with higher a "uniqueness" value - the negative of average similarity of a term to all other terms. REVIGO was run under the configurations "allowed similarity: medium (C value=0.7)", "GO term size: Homo sapiens", and "semantic similarity measure: SimRel". REVIGO used GO annotations from the July 2015 monthly release by the GO consortium (go_201507-termdb.obo-xml.gz, GO consortium) and UniProt-to-GO mapping data from the EBI GOA project, downloaded at the $27^{\text {th }}$ July 2015 (gene_association.goa_uniprot.gz). The terms that remained in the table after the clustering procedure are the cluster representatives; no two representatives will be more similar than the cutoff value $\mathrm{C}$. The procedures were done for each functional category separately. 


\section{Activation likelihood estimation meta-analysis}

The meta-analyses were conducted using a revised version $[5,6]$ of the activation likelihood estimation (ALE) approach for coordinate-based meta-analysis of neuroimaging results [2729]. ALE is based upon the approach to treat the reported foci as centers of 3D Gaussian probability distributions reflecting the spatial uncertainty associated with each reported set of coordinates $[5,29]$ and to determine brain regions showing a convergence of findings across studies which is higher than expected under a spatially random spatial association. All reported foci for a given experiment were combined for each voxel to produce a modeled activation map (MA map [28]). ALE scores describing the convergence of coordinates for each location were then calculated via the union of individual MA maps. To distinguish areas where the convergence between studies was greater than it would be expected by chance (i.e. to separate true convergence from noise) ALE scores were compared to a nonlinear histogram integration based on the frequency of distinct MA values [6]. For statistical inference, the ensuing statistical parametric maps were then thresholded at $p<0.05$ (cluster-level FWE, corrected for multiple comparisons, cluster-forming threshold at voxel level $p<0.001$ [6].

Given the frequent claims regarding the effects of different gene variants on the same brain regions, we decided to conduct a first meta-analysis pooling across all studies that declared to report on schizophrenia susceptibility variants. In a subsequent step, we aimed to investigate, whether significant effects were detectable in subsamples defined by diagnostic status. We, thus, conducted meta-analyses for the following subgroups: healthy controls, schizophrenia patients, genotype $\mathrm{X}$ diagnosis interaction. Resulting clusters were analyzed to delineate, which coordinates contributed to a resulting cluster and the tagged information was extracted (see "Categorization of studies according to the diagnostic status"). We finally pooled over studies using biological information (see "Grouping of studies according to gene ontology").

We used the Anatomy Toolbox [30-32] to refer all obtained clusters to compare the localization of the significant effects to histological areas. 


\section{Conjunction analysis}

Brain structure changes have been robustly characterized in schizophrenia patients. To relate our findings to a neuroanatomical endophenotype of schizophrenia, we carried out a conjunction analysis between the clusters of our actual meta-analysis and the results of a previous meta-analysis of our lab on structural changes in schizophrenia [33]. In detail, we computed the intersection of the thresholded maps using FSLmaths [34]. 


\section{Results}

\section{Main analysis pooling over all studies}

We did not find any significant results in the meta-analysis for the main contrast that pooled the data from all 30 studies (best $p$-value $=.3$ ).

\section{Analyses according to the original contrasts}

Our meta-analysis on gene $\mathrm{x}$ diagnosis interactions yielded two significant clusters, the larger one in the left middle temporal gyrus (allocation by cytoarchitectonic probabilistic mapping: TE 1.0, extending into TE 1.2; $-51,-19,1, \mathrm{k}=98$ ) and a slightly smaller one in the right gyrus rectus close to the midline (mainly located in area Fo2; 9, 13, -23, k=86). Coordinates that contributed to the cluster in the left middle temporal gyrus stemmed from contrasts of studies investigating genetic variants in the following genes: ZNF804A (rs1344706), CNNM2 (rs7914558), AKT1 (rs2494732) and DTNBP1 (rs1011313). For the cluster in the right supramarginal gyrus, we found coordinates contributing that investigated variants in the following genes: VAV3 (rs1410403), CCDC68 (rs12966547) and DISC1 (rs821597); (Figure 1, Table 2). We did not find significant results in the meta-analyses for any other contrast.

To gain insight into possible biological relationships between the seven implicated genes, we mapped six of them to $266 \mathrm{GO}$ terms of the category "biological process" (AKT1, CNNM2, DISC1, DTNBP1, VAV3), then five to 59 "cellular component" terms (AKT1, CNNM2, DISC1, $D T N B P 1, V A V 3)$, and three to 17 "molecular function" terms (AKT1, VAV3, ZNF804A); CCDC68 was not mapped to any category due to missing data in the bioinformatical resource that we used. To reduce the redundancy among the mapped data, we performed clustering analyses and found representative, non-redundant subsets of semantically similiar terms; this procedure was done for each category separately. For "biological process", we identified 154 cluster representatives, 40 for "cellular component", and 16 for "molecular function" (Supplementary Table 3). 


\section{Analyses according to gene ontology}

To achieve a data-driven grouping for the coordinate-based meta-analyses, we mapped the 26 genes from the selected literature to GO functional categories and performed a GSEA in each category. We found the terms "Axon", "cellular prohection", "neuron part", "neuron projection", "somatodendritic compartment" and "regulation of protein modification process".

Overall, we did not observe any significant result in the coordinate-based meta-analyses that were informed by gene ontology.

Conjunction analysis between the genotype $X$ diagnosis finding and structural changes in schizophrenia

There was no spatial overlap between our present findings and the clusters that have been identified by our previous meta-analysis on brain structure changes in schizophrenia [33]. 


\section{Discussion}

Imaging genetics approaches have been discussed critically, since candidate gene associations with brain function have generally not been confirmed in meta-analyses [35]. The lack of significant findings in our main meta-analysis and most of our sub-analyses are well in accordance with this notion.

It deserves to be emphasized that we only retrieved significant results, when we pooled over studies analyzing gene $X$ diagnosis interactions, while other groupings, e.g., based on biological information about the implicated genes (GO terms) did not yield any significant convergence of findings. The latter finding appears as contradictory to the prevalent notion in the field that research approaches based on biological (patho-)mechanisms might help to reduce the heterogeneity of imaging findings compared to a purely symptoms-based definition of schizophrenia [21,22].

\section{Methodological considerations}

There are several caveats that limit the interpretation of our results. First, our approach quantitatively summarizes the findings of structural imaging studies across susceptibility gene variants. While we purposefully aimed to include all variants that were described by the authors of the respective study themselves as associated with schizophrenia to avoid an early selection bias, our data pool is certainly limited to the published literature. Consequently, a GWAS that does not entail such limitation might yield different results regarding a potential homogenization of study findings on structural changes due to biological pathways.

Second, we here utilized an observer-independent approach for a grouping of studies due to biological mechanisms, including a network analysis of the protein encoding genes and a subsequent enrichment analysis available through STRING v10 [24]. This approach yields the great advantage of a systematic data-driven grouping, but is certainly limited by the current characterization of the respective gene products. This is especially important with regard to 
genes identified by actual GWAS approaches [3] that have attracted increased attention only recently after their identification, rendering their biological functions often as comparatively sparsely understood. Thus, our GO term analysis might favor an enrichment of longer known and therefore better characterized genes, despite lesser evidence for their association with schizophrenia. Moreover, many classical candidate genes were not found to be associated with schizophrenia by GWAS, which may create more biological heterogeneity. This may distort the results of our subsequent ALE analysis.

Third, the number of studies enrolled in the genotype $X$ diagnosis is rather low $(n=15)$. While certainly a higher number of contributing studies would be desirable [36], it should be emphasized that ALE-based meta-analyses yielded significant and robust results enrolling even smaller numbers of studies [37].

While ALE is a well validated and widely used coordinate-based meta-analytic approach [6], a mega-analysis pooling over original imaging data sets may have most likely provided a greater sensitivity. However, it should also be noted that this advantage of a mega-analysis would have come at the prize of a limited generalization, since it would not pool across different analysis approaches.

Moreover, to avoid subjective selection biases, we have pooled over all studies on genetic variants that were classified by the authors of the respective original studies as associated with schizophrenia. Given the recent progress in this field, it stands to reason that a meta-analysis on studies analyzing only variants with GWAS evidence might be a very promising approach. Such an approach would also allow to differentiate between protective and risk alleles and offer the option of respective subanalyses. However, the number of imaging genetics studies on GWAS variants is insufficient up to now to allow a robust analysis. Important insights into the pathogenesis of brain structure changes in schizophrenia can be expected, once a sufficiently high number of studies is available. 
Grouping of studies due to gene $X$ diagnosis interactions, not biological information, leads to significant results

Diagnostic schemes based solely on a constellation of symptoms have been criticized because of their poor reliability in clinical settings [38], their instability over the course of time and their low specificity (summarized in [21]). The two major diagnostic systems used today, ICD-1021 and $\mathrm{DSM}-\mathrm{V}^{20}$, however, both base their diagnostic criteria for schizophrenia on a catalogue of symptoms and demand that a certain number out of this pool must to be present over a given period of time. It seems proximate that this syndromal definition might create a significant biological heterogeneity. In other words: the concept of schizophrenia might serve only as an umbrella that envelopes a group of etiologically and pathophysiologically heterogeneous disorders. Correspondingly, the frequent finding of a greater heterogeneity within patient groups compared to healthy controls by neuroimaging studies has been interpreted as indicator that different endophenotypes are all subsumed under the label of schizophrenia[21]. This has led researchers to question the validity of the concept of schizophrenia and raised the demand to move research away from cohorts defined by diagnostic criteria towards dimensions based on clinical phenomenology and putative biological underpinnings.

Imaging genetics approaches have been proposed to delineate distinct endophenotypes and, thus, reduce heterogeneity within the patient group [cf. 7]. While certainly a deconstruction of schizophrenia itself is not possible by our approach, it does allow proxy conclusions, whether a grouping of imaging genetics studies along biological criteria helps to increase the likelihood of convergent findings. An increased likelihood of convergent findings under these conditions, in turn, would be an indicator for distinct endophenotypes.

We here capitalized on the richness of the imaging genetics literature to meta-analytically compare the spatial convergence of findings reported by original studies after grouping them due biological mechanisms or due primarily non-biologically derived criteria. Given the above mentioned considerations, we hypothesized that a grouping by biological mechanisms would lead to a stronger convergence of results than those based on other criteria. To our surprise, 
we found significant results only in our meta-analysis on studies reporting on genotype $X$ diagnosis interactions, while the grouping of studies based on biological mechanisms did not yield any significant results.

Given these findings, schizophrenia as defined by today's classification systems might be biologically a more homogeneous entity than the currently prevalent notion indicates. Interactions between genetic risk variants and general disease processes could play a decisive role in the actual manifestation of brain structure changes, while the effects of genetic risk variants in healthy subjects might only exert minor effects. This idea of a critical effect of the manifestation of disease on genotype effects is supported by the time course of brain structure changes before and after the onset of schizophrenia. Although mild differences in brain structure between asymptomatic subjects at high risk for psychosis and typical controls can be observed, it is transition to psychosis that goes along with pronounced changes in brain structure, especially with volume loss in frontal and temporal brain regions ${ }^{43}$. Also after the manifestation of the disorder, structural pathologies remain progressive, especially during the first year [40], and are associated with psychopathological dimensions [41]. These progressive brain structure changes, obviously closely linked to the onset and/or worsening of symptoms, indicate that disease-associated processes are important modulators of brain structure changes in schizophrenia patients. According to our results, this modulator might play a crucial role in mediating effects of gene variants on brain structure. The finding that grouping according to diagnostic status indeed leads to significant results, while a sorting by biological mechanisms fails to do so, could be a valid indicator that - despite the common notion - the diagnostic criteria for schizophrenia create a neurobiologically comparatively homogeneous group of patients, at least with regard to brain structure changes.

Analysis of studies on gene $X$ diagnosis interactions reveals a fronto-temporal pattern of brain structure changes 
The larger cluster that we retrieved for our genotype $X$ diagnosis meta-analysis was located in the left middle temporal gyrus. Analysis by cytoarchitectural probabilistic mapping referred the changes to be mainly located in TE1.0, partially extending into TE1.2, both being cytoarchitectonic subdivisions of the primary auditory cortex [42]. Auditory hallucinations are a hallmark in schizophrenia symptomatology and have been highlighted as a key symptom of the disorder in the literature early on [43]. Functional imaging studies have pinpointed the primary auditory cortex as one of the hubs involved in the pathogenesis of auditory hallucinations [44]. Concordant with these findings, on a structural level, also schizophrenia patients with auditory hallucinations showed convergent evidence for grey matter atrophy in the primary auditory corte [45]. Our finding of convergent brain structure changes associated with schizophrenia risk variants in the primary auditory cortex provides a potential explanatory model for the influence of genetic factors on this brain region that is a pivotal intersection in the pathophysiology of auditory hallucinations.

Previous findings hint at an association between volume changes of the orbitofrontal cortex and cognitive deficits [46]. Complementary to this, also abnormal structural connectivity has been linked to impaired cognitive functioning in schizophrenia patient [47]. Cognitive functioning in schizophrenia patients, in turn, has been shown to be amongst the symptom domains with the highest heritability for this disorder [48]. The results of our study suggest that the orbitofrontal cortex might be a critical hub, in which genetic variants might exert an influence on psychopathological dimensions, namely cognitive functioning, by modulating brain structure.

Remarkably, the clusters retrieved from our analysis showed no overlap with regions that were consistently structurally altered in schizophrenia patients [37]. This finding seems to be counterintuitive at first, though, might be potentially explained by the fact that the structural endophenotype of schizophrenia that we used here in our analysis was derived from a previous meta-analysis. Meta-analyses have the potential to determine a "smallest common denominator" of findings that converge across studies. However, they certainly only have a limited potential to capture interindividual variances. With regard to schizophrenia, 
interindividual variances have been linked to different clinical presentations. Grey matter reductions, e.g., in various regions of the right lateral temporal lobe and in the right anterior cingulate cortex have been reported to be associated with increased symptom severity in psychopathological dimensions, such as formal thought disorder and paranoia [49]. In another sample, a correlation between grey matter volume and working memory performance was found in schizophrenia patients [50]. Also hints at an association between reduced cortical thickness of the right temporo-parietal junction and persisting negative symptoms [51]. It should be noted that all these regions implicated in psychopathological variance are situated outside the network we found to be altered in our study on brain structure changes in schizophrenia [37]. Following this line of thought, the genes contributing to the clusters found by our analysis might contribute to the manifestation of these interindividual differences of brain structural changes in schizophrenia patients.

Biological roles of the genes that contributed to the clusters found by our gene $X$ diagnosis interactions analysis

We used post hoc bioinformatical analyses of the genes that contributed to the two clusters of our gene $\mathrm{X}$ diagnosis interactions analysis to reduce the redundancy among the GO terms for a better biological interpretation of this finding. Multiple GO terms that are representative for clusters of semantically similar terms highlight biological processes obviously involved in the central nervous system, especially the cluster representative brain development (GO:0007420) including proliferation and differentiation of neuronal cells and formation of macroanatomical structures like forebrain, pallium and telencephalon (AKT1, DTNBP1, VAV3, DISC1) [52-55]. Further aspects at the cellular and tissue level of the brain are accentuated through cluster representatives such as gliogenesis (GO:0042063; AKT1, DISC1) [56,57], Schwann cell differentiation (GO:0014037; AKT1) [58] and anatomical structure formation involved in morphogenesis (GO:0048646) that emphasizes axon guidance (DISC1, DTNBP1, VAV3) [59-61]. 
Many representatives belong to neurotransmission and signaling pathways strongly discussed in the context of schizophrenia, of note, regulation of dopamine receptor signaling pathway (GO:0060159; DTNBP1) [62], G-protein coupled receptor signaling pathway (GO:0007186; AKT1) [63], canonical Wnt signaling pathway (GO:0060070; DISC1) [64], TOR signaling (GO:0031929; DISC1) [63], and regulation of kinase activity including JNK and JUN (GO:0043549; AKT1, DTNBP1, VAV3) [65,66]. The representative term positive regulation of lymphocyte activation (GO:0051251; AKT1, VAV3) $[67,68]$ stresses a role of the immune system in disease etiology. The representative metal ion binding (GO:0046872) addresses the molecular function of ZNF804A to form a complex with $\mathrm{Zn}^{2+}$ ions for binding to DNA. A recent study has provided strong evidence that ZNF804A regulates the expression of four schizophrenia-associated genes of which two (COMT, DRD2) have been contributed to our gene $x$ diagnosis interaction finding [69]. Moreover, ZNF804A is the only of the seven genes that harbours a SNP showing genome-wide significant association with schizophrenia [3].

Last but not least, our results might support two hypotheses that are often debated in schizophrenia research: The comorbidity between schizophrenia and heart disease by the representative term cardiovascular system development (GO:0072358) that might go beyond antipsychotics-associated effects (AKT1, VAV3) [70,71], the representatives sexual reproduction and reproductive system development (GO:0019953, GO:0061458; AKT1) [72] indicate a possible link to reduced fecundity in the biology of schizophrenia [73].

\section{$\underline{\text { Conclusions }}$}

We here have demonstrated that a large-scale coordinate-based meta-analysis can be meaningfully used to synthesize findings of structural imaging genetics studies pooling over different schizophrenia susceptibility variants. It deserves to be emphasized that the large majority of our contrasts, including the main contrast pooling over all studies, did not yield significant results. This lack of significant findings for most contrasts applied should urge a more cautious interpretation of the results of individual imaging genetics studies. Despite this 
majority of negative findings, remarkably, we found a significant convergence of results for studies analyzing genotype $X$ diagnosis interactions in the left middle temporal gyrus and the right gyrus rectus close to the midline, both regions that have been implicated previously by studies as potentially involved in the pathophysiology of schizophrenia. This is the first metaanalytical evidence for a convergence of results in imaging genetics. Finally, our data provides first hints that schizophrenia as defined by might be a more homogeneous and biologically valid entity than commonly perceived.

\section{Acknowlegements}

T.N.-J. was supported by the Deutsche Forschungsgemeinschaft (DFG). 


\section{Conflict of interest}

The authors declare that there is no conflict of interest.

\section{References}


1. Egan MF, Goldberg TE, Kolachana BS, Callicott JH, Mazzanti CM, Straub RE et al. Effect of COMT Val108/158 Met genotype on frontal lobe function and risk for schizophrenia. Proc Natl Acad Sci U S A. 2001; 98(12): 6917-6922.

2. Mier D, Kirsch P, Meyer-Lindenberg A. Neural substrates of pleiotropic action of genetic variation in COMT: a meta-analysis. Mol Psychiatry. 2010; 15(9): 918-927.

3. Schizophrenia Working Group of the Psychiatric Genomics consortium. Biological insights from 108 schizophrenia-associated genetic loci. Nature. 2014; 511(7510): 421-427.

4. Nickl-Jockschat T, Janouschek H, Eickhoff SB, Eickhoff CR. Lack of meta-analytic evidence for an impact of COMT Val158Met genotype on brain activation during working memory tasks [published online February 28, 2015]. Biol Psychiatry. doi: 10.1016/j.biopsych.2015.02.030

5. Eickhoff SB, Laird AR, Grefkes C, Wang LE, Zilles K, Fox PT. Coordinate-based ALE metaanalysis of neuroimaging data: A random-effects approach based on empirical estimates of spatial uncertainty. Hum Brain Mapp. 2009; 30(9): 2907-2926.

6. Eickhoff SB, Bzdok D, Laird AR, Kurth F, Fox PT. Activation Likelihood Estimation metaanalysis revisited. Neurolmage. 2012; 59(3): 2349-2361.

7. Thompson PM, Stein JL, Medland SE, Hibar DP, Vasquez AA, Renteria ME et al. The ENIGMA Consortium: large-scale collaborative analyses of neuroimaging and genetic data. Brain Imaging Behav. 2014; 8(2):153-182.

8. Barnes A, Isohanni M, Barnett JH, Pietiläinen O, Veijola J, Miettunen J, et al. Neuregulin1 genotype is associated with structural differences in the normal human brain. Neuroimage. 2012; 59 (3): 2057-2061.

9. Cassidy C, Buchy L, Bodnar M, Dell'elce J, Choudhry Z, Fathalli F, et al. Association of a risk allele of ANK3 with cognitive performance and cortical thickness in patients with firstepisode psychosis. J Psychiatry Neurosci. 2014; 39(1): 31-39. 
10. Donohoe G, Rose E, Frodl T, Morris D, Spoletini I, Adriano F et al. ZNF804A risk allele is associated with relatively intact gray matter volume in patients with schizophrenia. Neuroimage. 2011; 54(3): 2132-2137.

11. Kempf L, Nicodemus KK, Kolachana B, Vakkalanka R, Verchinski BA, Egan MF et al. Functional polymorphisms in $\mathrm{PRODH}$ are associated with risk and protection for schizophrenia and fronto-striatal structure and function [published online November 7, 2008]. PLoS Genet. doi: 10.1371/journal.pgen.1000252

12. Nenadic I, Maitra R, Scherpiet S, Gaser C, Schultz CC, Schachtzabel C et al. Glutamate receptor $\delta 1$ (GRID1) genetic variation and brain structure in schizophrenia. J Psychiatr Res. 2012; 46(12): 1531-1539.

13. Ho BC, Milev P, O'Leary DS, Librant A, Andreasen NC, Wassink TH. Cognitive and magnetic resonance imaging brain morphometric correlates of brain-derived neurotrophic factor Val66Met gene polymorphism in patients with schizophrenia and healthy volunteers. Arch Gen Psychiatry. 2006; 63(7): 731-740.

14. Nenadic I, Maitra R, Basmanav FB, Schultz CC, Lorenz C, Schachtzabel C et al. ZNF804A genetic variation (rs1344706) affects brain grey but not white matter in schizophrenia and healthy subjects. Psychol Med. 2015; 45(1): 143-152.

15. Ohi K, Hashimoto R, Yasuda Y, Nemoto K, Ohnishi T, Fukumoto M et al. Impact of the genome wide supported NRGN gene on anterior cingulate morphology in schizophrenia [published online January 12, 2012]. PLoS One. doi: 10.1371/journal.pone.0029780

16. Mathieson I, Munafò MR, Flint J. Meta-analysis indicates that common variants at the DISC1 locus are not associated with schizophrenia. Mol Psychiatry 2012; 17(6): 634-641.

17. Okochi T, Ikeda M, Kishi T, Kawashima K, Kinoshita Y, Kitajima T et al. Meta-analysis of association between genetic variants in COMT and schizophrenia: an update. Schizophr Res 2009; 110(1-3): 140-148. 
18. Franke B, Stein JL, Ripke S, Anttila V, Hibar DP, van Hulzen KJ et al. Genetic influences on schizophrenia and subcortical brain volumes: large-scale proof of concept. Nat Neurosc. 2016. 19(3): 420-431.

19. American Psychiatric Association. Diagnostic and statistical manual of mental disorders (5th ed.). Washington, DC, USA, 2013.

20. World Health Organization. International Statistical Classification of Diseases and Related Health Problems. (2010). (http://www.who.int/classifications/icd/en/bluebook.pdf?ua=1; retrieved March 07th, 2016).

21. Tandon R, Gaebel W, Barch DM, Bustillo J, Gur RE, Heckers S et al. Definition and description of schizophrenia in the DSM-5. Schizophr Res. 2013; 150(1): 3-10.

22. Gur RE, Keshavan MS, Lawrie SM. Deconstructing psychosis with human brain imaging. Schizophr Bull. 2007; 33(4): 921-931.

23. Gray KA, Daugherty LC, Gordon SM, Seal RL, Wright MW, Bruford EA. Genenames.org: the HGNC resources in 2013. Nucleic Acids Res. 2013; 41(Database issue): D545-52.

24. Szklarczyk D, Franceschini A, Wyder S, Forslund K, Heller D, Huerta-Cepas J et al. STRING v10: protein-protein interaction networks, integrated over the tree of life. Nucleic Acids Res. 2015; 43: D447-D452.

25. Supek F, Bošnjak M, Škunca N, Šmuc T. REVIGO summarizes and visualizes long lists of gene ontology terms. PLoS One 2011; 6: e21800.

26. Pesquita C, Faria D, Falcão AO, Lord P, Couto FM. Semantic similarity in biomedical ontologies. PLoS Comput Biol 2009; 5: e1000443.

27. Laird AR, Fox PM, Price CJ, Glahn DC, Uecker AM, Lancaster JL et al. ALE meta-analysis: controlling the false discovery rate and performing statistical contrasts. Hum Brain Mapp. 2005; 25:155-164 
28. Turkeltaub PE, Eden GF, Jones KM, Zeffiro TA. Meta-analysis of the functional neuroanatomy of single-word reading: method and validation. Neurolmage. 2002; 16: 765780

29. Turkeltaub PE, Eickhoff SB, Laird AR, Fox M, Wiener M, Fox P. Minimizing withinexperiment and within-group effects in Activation Likelihood Estimation meta-analyses. Hum Brain Mapp. 2012; 33(1): 1-13

30. Eickhoff SB, Stephan KE, Mohlberg H, Grefkes C, Fink GR, Amunts K, Zilles K. A new SPM toolbox for combining probabilistic cytoarchitectonic maps and functional imaging data. Neurolmage. 2005; 25: 1325-1335.

31. Eickhoff SB, Heim S, Zilles K, Amunts K. Testing anatomically specified hypotheses in functional imaging using cytoarchitectonic maps. Neurolmage. 2006; 32: 570-582.

32. Eickhoff SB, Paus T, Caspers S, Grosbras MH, Evans AC, Zilles K, Amunts K. Assignment of functional activations to probabilistic cytoarchitectonic areas revisited. Neurolmage. 2007; 36(3): 511-521.

33. Nickl-Jockschat T, Schneider F, Pagel AD, Laird AR, Fox PT, Eickhoff SB. Progressive pathology is functionally linked to the domains of language and emotion: meta-analysis of brain structure changes in schizophrenia patients. Eur Arch Psychiatry Clin Neurosci 2011; 261 Suppl 2: S166-171.

34. Jenkinson M, Beckmann CF, Behrens TE, Woolrich MW, Smith SM. FSL. Neuroimage. 2012; 62(2): 782-790.

35. Poldrack RA, Farah MJ. Progress and challenges in probing the human brain. Nature. 2015; 526(7573): 371-379.

36. Eickhoff SB, Nichols TE, Laird AR, Hoffstaedter F, Amunts K, Fox PT et al. Behavior, sensitivity, and power of activation likelihood estimation characterized by massive empirical simulation. Neurolmage. 2016; 137: 70-85. 
37. Nickl-Jockschat T, Rottschy C, Thommes J, Schneider F, Laird AR, Fox PT, Eickhoff SB. Neural networks related to dysfunctional face processing in autism spectrum disorder. Brain Struct Funct. 2015; 220(4): 2355-2371.

38. Miller PR, Dasher R, Collins R, Griffiths P, Brown F. Inpatient diagnostic assessments: 1. Accuracy of structured vs. unstructured interviews. Psychiatry Res. 2001; 105(3): 255-264.

39. Fusar-Poli P, Radua J, McGuire P, Borgwardt S. Neuroanatomical maps of psychosis onset: voxel-wise meta-analysis of antipsychotic-naive VBM studies. Schizophr Bull. 2012; 38(6): 1297-1307.

40. Andreasen NC, Nopoulos P, Magnotta V, Pierson R, Ziebell S, Ho BC. Progressive brain change in schizophrenia: a prospective longitudinal study of first-episode schizophrenia. Biol Psychiatry. 2011; 70(7): 672-679.

41. Ho BC, Andreasen NC, Nopoulos P, Arndt S, Magnotta V, Flaum M. Progressive structural brain abnormalities and their relationship to clinical outcome: a longitudinal magnetic resonance imaging study early in schizophrenia. Arch Gen Psychiatry. 2003; 60 (6): 58594.

42. Morosan P, Rademacher J, Schleicher A, Amunts K, Schormann T, Zilles K. Human primary auditory cortex: cytoarchitectonic subdivisions and mapping into a spatial reference system. Neuroimage. 2001; 13(4): 684-701.

43. Schneider, K. Klinische Psychopathologie. Thieme: Stuttgart, Germany,1950.

44. Jardri R, Pouchet A, Pins D, Thomas P. Cortical activations during auditory verbal hallucinations in schizophrenia: a coordinate-based meta-analysis. Am J Psychiatry. 2011; 168(1): 73-81.

45. Modinos G, Costafreda SG, van Tol MJ, McGuire PK, Aleman A, Allen P. Neuroanatomy of auditory verbal hallucinations in schizophrenia: a quantitative meta-analysis of voxelbased morphometry studies. Cortex. 2013; 49(4): 1046-1055. 
46. Nakamura M, Nestor PG, Levitt JJ, Cohen AS, Kawashima T, Shenton ME, McCarley RW. Orbitofrontal volume deficit in schizophrenia and thought disorder. Brain. 2008; 131(Pt 1): 180-195.

47. James A, Joyce E, Lunn D, Hough M, Kenny L, Ghataorhe P et al. Abnormal frontostriatal connectivity in adolescent-onset schizophrenia and its relationship to cognitive functioning. Eur Psychiatry. 2016; 35: 32-38.

48. van Os J, Kenis G, Rutten BP. The environment and schizophrenia. Nature. 2010; 468(7321): 203-212.

49. Lui S, Deng W, Huang X, Jiang L, Ma X, Chen H et al. Association of cerebral deficits with clinical symptoms in antipsychotic-naive first-episode schizophrenia: an optimized voxelbased morphometry and resting state functional connectivity study. Am J Psychiatry. 2009; 166(2): 196-205.

50. Zierhut KC, Schulte-Kemna A, Kaufmann J, Steiner J, Bogerts B, Schiltz K. Distinct structural alterations independently contributing to working memory deficits and symptomatology in paranoid schizophrenia. Cortex. 2013; 49(4): 1063-1072.

51. Bodnar M, Hovington CL, Buchy L, Malla AK, Joober R, Lepage M. Cortical thinning in temporo-parietal junction (TPJ) in non-affective first-episode of psychosis patients with persistent negative symptoms [published online June 30, 2014]. PLoS One. doi: 10.1371/journal.pone.0101372

52. Zheng W, Wang H, Zeng Z, Lin J, Little PJ, Srivastava LK et al. The possible role of the Akt signaling pathway in schizophrenia. Brain Res. 2012; 1470: 145-158.

53. Xu Y, Sun Y, Ye H, Zhu L, Liu J, Wu X et al. Increased dysbindin-1B isoform expression in schizophrenia and its propensity in aggresome formation. Cell Discov. 2015; 1: 15032.

54. Aleksic B, Kushima I, Hashimoto R, Ohi K, Ikeda M, Yoshimi A et al. Analysis of the VAV3 as candidate gene for schizophrenia: Evidences from voxel-based morphometry and mutation screening. Schizophr Bull. 2013; 39(3): 720-728. 
55. Johnstone M, Thomson PA, Hall J, McIntosh AM, Lawrie SM, Porteous DJ. DISC1 in schizophrenia: Genetic mouse models and human genomic imaging. Schizophr Bull. 2011; 37(1): 14-20.

56. Lee DY, Yeh TH, Emnett RJ, White CR, Gutmann DH. Neurofibromatosis-1 regulates neuroglial progenitor proliferation and glial differentiation in a brain region-specific manner. Genes Dev. 2010; 24(20): 2317-2329.

57. Wang S, Liang Q, Qiao H, Li H, Shen T, Ji F et al. DISC1 regulates astrogenesis in the embryonic brain via modulation of RAS/MEK/ERK signaling through RASSF7. Development. 2016; 143(15): 2732-2740.

58. Taveggia C, Feltri ML, Wrabetz L. Signals to promote myelin formation and repair. Nat Rev Neurol. 2010; 6(5): 276-287.

59. Kvajo M, McKellar H, Drew LJ, Lepagnol-Bestel A-M, Xiao L, Levy RJ et al. Altered axonal targeting and short-term plasticity in the hippocampus of Disc1 mutant mice [published online November 2, 2011]. Proc Natl Acad Sci U S A. doi: 10.1073/pnas.1114113108

60. Lee SA, Kim SM, Suh BK, Sun HY, Park YU, Hong JH et al. Disrupted-in-schizophrenia 1 (DISC1) regulates dysbindin function by enhancing its stability. J Biol Chem. 2015; 290(11): 7087-7096.

61. Cowan CW, Shao YR, Sahin M, Shamah SM, Lin MZ, Greer PL et al. Vav family GEFs link activated Ephs to endocytosis and axon guidance. Neuron. 2005; 46(2): 205-17.

62. Papaleo F, Weinberger DR. Dysbindin and schizophrenia: It's dopamine and glutamate all over again. Biol Psychiatry. 2011; 69(1): 2-4.

63. New DC, Wu K, Kwok AWS, Wong YH. G protein-coupled receptor-induced Akt activity in cellular proliferation and apoptosis. FEBS J. 2007; 274(23): 6025-6036. 
64. Salinas PC. Wnt signaling in the vertebrate central nervous system: From axon guidance to synaptic function [published online February 1, 1012]. Cold Spring Harb Perspect Biol. doi: 10.1101/cshperspect.a008003

65. Kim AH, Yano H, Cho H, Meyer D, Monks B, Margolis B et al. Akt1 regulates a JNK scaffold during excitotoxic apoptosis. Neuron. 2002; 35: 697-709.

66. Tohyama M, Miyata S, Hattori T, Shimizu S, Matsuzaki S. Molecular basis of major psychiatric diseases such as schizophrenia and depression. Anat Sci Int. 2015; 90(3): $137-$ 143.

67. Bitanihirwe BKY, Weber L, Feldon J, Meyer U. Cognitive impairment following prenatal immune challenge in mice correlates with prefrontal cortical AKT1 deficiency. Int $\mathrm{J}$ Neuropsychopharmacol. 2010; 13: 981-996.

68. Inabe K, Ishiai M, Scharenberg AM, Freshney N, Downward J, Kurosaki T. Vav3 Modulates B Cell Receptor Responses by Regulating Phosphoinositide 3-Kinase Activation. J Exp Med. 2002; 11891200: 189-200.

69. Girgenti MJ, LoTurco JJ, Maher BJ. ZNF804a regulates expression of the schizophreniaassociated genes PRSS16, COMT, PDE4B, and DRD2 [published online February 27, 2012]. PLoS One. 2012; doi: 10.1371/journal.pone.0032404

70. Emamian ES. AKT/GSK3 signaling pathway and schizophrenia. Front Mol Neurosci. 2012; 5: 33.

71. Sauzeau V, Horta-Junior JAC, Riolobos AS, Fernández G, Sevilla MA, Ló Pez DE et al. Vav3 Is Involved in GABAergic Axon Guidance Events Important for the Proper Function of Brainstem Neurons Controlling Cardiovascular, Respiratory, and Renal Parameters. Mol Biol Cell. 2010; 21: 4251-4263.

72. Kim ST, Omurtag K, Moley KH. Decreased spermatogenesis, fertility, and altered Slc2A expression in Akt1-/- and Akt2-/- testes and sperm. Reprod Sci. 2012; 19: 31-42. 
73. Haukka J, Suvisaari J, Lönnqvist J. Fertility of patients with schizophrenia, their siblings, and the general population: a cohort study from 1950 to 1959 in Finland. Am J Psychiatry. 2003; 160(3): 460-463.

74. Aleksic B, Kushima I, Hashimoto R, Ohi K, Ikeda M, Yoshimi A et al. Analysis of the VAV3 as candidate gene for schizophrenia: evidences from voxel-based morphometry and mutation screening. Schizophr Bull. 2013; 39(3): 720-728.

75. Benedetti F, Poletti S, Radaelli D, Bernasconi A, Cavallaro R, Falini A et al. Temporal lobe grey matter volume in schizophrenia is associated with a genetic polymorphism influencing glycogen synthase kinase 3- $\beta$ activity. Genes Brain Behav. 2010; 9(4): 365-371.

76. Buckholtz JW, Meyer-Lindenberg A, Honea RA, Straub RE, Pezawas L, Egan MF et al. Allelic variation in RGS4 impacts functional and structural connectivity in the human brain. J Neurosci. 2007; 27(7): 1584-1593.

77. Dannlowski U, Kugel H, Grotegerd D, Redlich R, Suchy J, Opel N et al. NCAN CrossDisorder Risk Variant Is Associated With Limbic Gray Matter Deficits in Healthy Subjects and Major Depression. Neuropsychopharmacology. 2015; 40(11): 2510-2516.

78. Donohoe G, Frodl T, Morris D, Spoletini I, Cannon DM, Cherubini A et al. Reduced occipital and prefrontal brain volumes in dysbindin-associated schizophrenia. Neuropsychopharmacology. 2010; 35(2): 368-373.

79. Eker C, Kitis Ö, Ozan E, Okur H, Donat Eker O, Ersoy MA et al. BDNF Gene Val66met Polymorphism Associated Grey Matter Changes in Human Brain. Bulletin of Clinical Psychopharmacology. 2005; 15(3): 104-111.

80. Kido M, Nakamura Y, Nemoto K, Takahashi T, Aleksic B, Furuichi A et al. The polymorphism of YWHAE, a gene encoding 14-3-3epsilon, and brain morphology in schizophrenia: a voxel-based morphometric study [published online August 8, 2014]. PLoS One. doi: 10.1371/journal.pone.0103571 
81. Lencz T, Szeszko PR, DeRosse P, Burdick KE, Bromet EJ, Bilder RM, Malhotra AK. A schizophrenia risk gene, ZNF804A, influences neuroanatomical and neurocognitive phenotypes. Neuropsychopharmacology. 2010; 35(11): 2284-2291.

82. McIntosh AM, Baig BJ, Hall J, Job D, Whalley HC, Lymer GK et al. Relationship of catecholO-methyltransferase variants to brain structure and function in a population at high risk of psychosis. Biol Psychiatry. 2007; 61(10): 1127-1134.

83. Meyer-Lindenberg A, Straub RE, Lipska BK, Verchinski BA, Goldberg T, Callicott JH et al. Genetic evidence implicating DARPP-32 in human frontostriatal structure, function, and cognition. J Clin Invest. 2007; 117(3): 672-682.

84. Montag C, Weber B, Jentgens E, Elger C, Reuter M. An epistasis effect of functional variants on the BDNF and DRD2 genes modulates gray matter volume of the anterior cingulate cortex in healthy humans. Neuropsychologia. 2010; 48 (4): 1016-1021.

85. Nemoto K, Ohnishi T, Mori T, Moriguchi Y, Hashimoto R, Asada T, Kunugi H. The Val66Met polymorphism of the brain-derived neurotrophic factor gene affects age-related brain morphology. Neurosci Lett. 2006; 397(1-2): 25-29.

86. Ohi K, Hashimoto R1, Yamamori H, Yasuda Y, Fujimoto M, Umeda-Yano S et al. The impact of the genome-wide supported variant in the cyclin M2 gene on gray matter morphology in schizophrenia. Behav Brain Funct. 2013; 9: 40.

87. Ohi K, Hashimoto R, Yasuda Y, Fukumoto M, Nemoto K, Ohnishi T et al. The AKT1 gene is associated with attention and brain morphology in schizophrenia. World $\mathrm{J}$ Biol Psychiatry. 2013; 14(2): 100-113.

88. Thirunavukkarasu P, Vijayakumari AA, John JP, Halahalli HN, Paul P, Sen S et al.. An exploratory association study of the influence of dysbindin and neuregulin polymorphisms on brain morphometry in patients with schizophrenia and healthy subjects from South India. Asian J Psychiatr. 2014; 10: 62-68.

89. Tian T, Qin W, Liu B, Wang D, Wang J, Jiang T, Yu C. Catechol-O-methyltransferase Val158Met polymorphism modulates gray matter volume and functional connectivity of the 
default mode network [published online October 16, 2013]. PLoS One. doi: 10.1371/journal.pone.0078697

90. Trost S, Platz B, Usher J, Scherk H, Wobrock T, Ekawardhani S et al. The DTNBP1 (dysbindin-1) gene variant rs2619522 is associated with variation of hippocampal and prefrontal grey matter volumes in humans. Eur Arch Psychiatry Clin Neurosci. 2013; 263 (1): 53-63.

91. Trost S, Platz B, Usher J, Scherk H, Wobrock T, Ekawardhani S et al. DISC1 (disruptedin-schizophrenia 1) is associated with cortical grey matter volumes in the human brain: a voxel-based morphometry (VBM) study. J Psychiatr Res. 2013; 47(2): 188-196.

92. Vijayakumari AA, John JP, Halahalli HN, Paul P, Thirunavukkarasu P, Purushottam M, Jain S. Effect of polymorphisms of three genes mediating monoamine signalling on brain morphometry in schizophrenia and healthy subjects. Clin Psychopharmacol Neurosci. 2015; 13(1): 68-82.

93. Wei Q, Diao F, Kang Z, Gan Z, Han Z, Zheng L et al. The effect of DISC1 on regional gray matter density of schizophrenia in Han Chinese population. Neurosci Lett. 2012; 517 (1): 21-24.

94. Zhang Y, Yan H, Tian L, Wang F, Lu T, Wang L et al. Association of MTHFR C677T polymorphism with schizophrenia and its effect on episodic memory and gray matter density in patients. Behav Brain Res. 2013; 243: 146-152.

95. Zinkstok J, Schmitz N, van Amelsvoort T, Moeton M, Baas F, Linszen D. Genetic variation in COMT and PRODH is associated with brain anatomy in patients with schizophrenia. Genes Brain Behav. 2008; 7(1): 61-69. 


\section{Figure 1}

Our meta-analysis on gene $\mathrm{x}$ diagnosis interactions yielded two significant clusters. One was located in the left middle temporal gyrus (allocation by cytoarchitectonic probabilistic mapping: TE 1.0, extending into TE 1.2; -51, -19, 1, k=98), while the second cluster was found in the right gyrus rectus close to the midline (mainly located in area Fo2; 9, 13, -23, k=86). Coordinates that contributed to the cluster in the left middle temporal gyrus stemmed from

contrasts of studies investigating genetic variants in the following genes: ZNF804A (rs1344706), CNNM2 (rs7914558), AKT1 (rs2494732) and DTNBP1 (rs1011313). For the cluster in the right supramarginal gyrus, we found coordinates contributing that investigated 
variants in the following genes: VAV3 (rs1410403), CCDC68 (rs12966547) and DISC1 (rs821597).

For statistical inference, the ensuing statistical parametric maps stemming from our ALE analysis were thresholded at $p<0.05$ (cluster-level FWE, corrected for multiple comparisons, cluster-forming threshold at voxel level $p<0.001^{29}$ ).

\section{Table 1}

Characteristics of the original studies contributing to our meta-analysis. A total of 30 studies met inclusion criteria. Table 1 summarizes the studies including the studied SNPs, genes mapping next to these SNPs, the original analyses and sample characteristics in the respective original studies.

\section{Table 2}

Summary of studies contributing to the significant clusters retrieved due to our meta-analysis on genotype $X$ diagnosis interactions. 


\section{Supplementary Table 1}

Combinations of search strings used and number of studies retrieved by the respective combinations in Pubmed (June 3rd 2015).

\section{Supplementary Table 2}

Overview over the symbols of the reported genes. These were checked if they match to the latest approved definition by the Human Gene Nomenclature Committee (HGNC; www.genenames.org). Disapproved symbols were substituted by the approved symbols. 
